\title{
CAFSM: A Communicating Adaptive Finite State Machine for Personalized Multimedia Streaming
}

\author{
Susan Elias \\ Dept. of Computer Science \\ Sri Venkateswara Coll of Engg \\ Sriperumbudur, Chennai \\ Tamil Nadu, India
}

\author{
Jasmin C.B \\ Dept. of Computer Science \\ Sri Venkateswara Coll of Engg \\ Sriperumbudur, Chennai \\ Tamil Nadu, India
}

\author{
Lisa Mathew \\ Dept. of Mathematics \\ Sri Venkateswara Coll of Engg \\ Sriperumbudur, Chennai \\ Tamil Nadu, India
}

\author{
Easwarakumar.K.S \\ Dept. of Computer Science \\ College of Engineering \\ Anna University, Chennai \\ Tamil Nadu, India
}

\author{
Richard Chbeir \\ Dept. of Computer Science \\ LE2I - Bourgogne University \\ Dijon, France
}

\begin{abstract}
In this paper we present the design of a multimedia presentation system which permits the dynamic adaptation of the content. The Communicating Adaptive Finite State Machine (CAFSM) presented in this paper, has been used to describe the multimedia streaming and presentation system proposed here. This system is driven by a set of messages that are used for communication and co-ordination among the various component machines which form the system
\end{abstract}

\section{Keywords}

Communicating, Adaptive, Finite State Machines, Multimedia Presentation, e-Learning

\section{INTRODUCTION AND MOTIVATION}

The Dynamic Extended Finite State Machine (DEFSM) was proposed in $[9,10]$ to model a multimedia synchronization and execution system. This presentation control model allows dynamic transitions in order to deal efficiently with user interactions thereby reducing the number of transitions required. Our attempt to modify the DEFSM model in order to present content that can be dynamically adapted, led to the development of the Communicating Adaptive Finite State Machine (CAFSM) presented here. The limitation of the DEFSM model is that each synchronization point in the presentation layout is modeled as a state in the DEFSM. Thus, the DEFSMs representing fast changing multimedia presentations will have several states and transitions. The number of states and transitions in our model are independent of the number of synchronization points in the presentation, thereby reducing the memory requirement of the system especially for fast changing multimedia presentations. Synchronization points are identified as points in the presentation layout where one or more media objects begin or end their presentation.

Communicating Finite State Machines (CFSM) were introduced in [2]. In this model Finite State Machines were used to model processes and queues to represent the communication channels between them. However the communication performed by the CFSM was carried out using a broadcast model while our proposed CAFSM model can also multicast data. In our proposed system the various components communicate with each other by exchanging a finite set of messages which have been specified here. The motivation behind this work was the need to develop a distributed multimedia presentation system that would ensure the synchronized playout, while simultaneously handling adaptation and user interactions efficiently. Other existing synchronisation models and their features are discussed in $[1,15,16]$.

\section{APPLICATION OF THE PROPOSED RESEARCH WORK}

This paper presents the design of a presentation tool that can support the dynamic adaptation of the contents in distributed multimedia applications. In this subsection a domain specific application of the adaptive multimedia presentation tool is presented.

\section{Adaptive E-Learning System:}

E-learning systems do exists and are successfully being utilized by several organizations and universities but the challenge now is to personalize the content for each user automatically. The motivation behind personalising content was the need to provide a learning environment to suit each person's learning capabilities. Adaptive eLearning systems are currently being designed and several existing elearning tools are being incorporated with this feature $[4,13,5]$. But most of them use predefined or adaptive tests [14] to decide the learning path, making them static adaptation models. Understanding learning models $[3,7]$ and incorporating them into e-learning systems is the biggest challenge and several research works have been focusing on this aspect. The integration of neuro-fuzzy logic [11] and eye tracking approaches $[8,12]$, to decide on the learning paths are currently being researched in order to dynamically adapt the content without the learners knowledge. 'Advance Personalised Learning' is also one of the 14 Grand Engineering Challenges for the 21st century put forth by the National Academy of Engineers [17].

In case of complex multimedia presentations where the data reside in distributed servers, the response time of the presentation tool is also important during adaptation. Presentation tools need to be redesigned to handle adaptation. An innovative model called Communicating Adaptive Finite State Machines CAFSM, introduced in this paper in the following section, is used to present the design of the proposed presentation tool. Quality of Experience $(Q o E)$ parameters are also being defined and used to evaluate the performance of Adaptive elearning Systems being designed. SCORM the standard used in the existing e-learning systems has also been extended [18] to support Adaptive e-learning. 


\section{COMMUNICATING ADAPTIVE FINITE STATE MACHINES: CAFSM}

This section presents the proposed synchronization mechanism for distributed multimedia presentations. In order to model the synchroniser we define a modification of the finite state machine and refer to it as Communicating Adaptive Finite State Machine (CAFSM). The proposed model uses three variations of the CAFSM referred to as
i)
ii) Media Schedule Managers (MSM) and
iii) Layout generator (LG)

The PFSM represents the entire presentation at the server $\left(P F S M_{\text {Server }}\right)$ and also at the client $\left(P F S M_{\text {Client }}\right)$. Media Schedule Managers (MSM) are used to co-ordinate the transfer/streaming of discrete/continuous media objects, and are present at both the server $M S M_{\text {server }}$ and at the client $M S M_{\text {client }}$. There is one $M S M$ for each continuous media object (such as video, audio and animation) present at the server as well as the client and a single $M S M$ each at the server and at the client to handle the transfer of all discrete media objects (such as text, images). The function of $L G$ is, to extract from the temporal layout of the presentation the list of media objects beginning and ending at each synchronization point, and communicate them to the PFSM client on demand. The communication between the various components of the synchronization model is depicted in Figure 1. To ensure synchronisation during playout, messages are used to control the entire data flow required for the presentation. The sequence of messages passed between the finite state machines and their state changes, as well as the details of the individual components of the proposed CAFSM model will be explained in the following subsections.

\subsection{Presentation Finite State Machines: PFSM}

Formally, a presentation finite state machine PFSM can be defined as a 4-tuple $(S, \Sigma, L, \delta)$, where $S$ is a finite set of states $\left\{\mathrm{S}_{1}, \mathrm{~S}_{2}, \mathrm{~S}_{3}\right\}, \sum$ is a set of the form $(m, M)$ where $m$ indicates a message and $M$ indicates the $C A F S M$ involved, $L$ is a set of participating media objects, and $\delta: S \times$ $2^{\Sigma} \times L^{2} \rightarrow S \times 2^{\Sigma} \times L^{2}$ is a transition function. The set of messages used by all the CAFSMs is given in Table 1. The transitions are in the form $\delta\left(S_{i},\left(m_{j}, M_{k}\right), L^{\prime}, L^{\prime \prime}\right)=\left(S_{l},\left(m_{p}, M_{q}\right), L^{*}, L^{* *}\right)$, where $S_{i}$ and $S_{l}$ are states of the PFSM under consideration, $\left(m_{j}, M_{k}\right)$ and $\left(m_{p}, M_{q}\right)$ are the messages received and sent respectively, along with the source / destination finite state machines, $L^{\prime} L^{\prime \prime}, L^{*}$ and $L^{* *}$ are lists of multimedia objects. Here, it is assumed that the PFSMs can communicate with each other as well as with the local MSMs only. In case there is a need to send or receive the same message $m$ to / from a list $L$ of local MSMs, the notation $M S M(L)$ is used in place of $M_{k}$ or $M_{q}$. All interactions from the user are treated as messages initiated by the

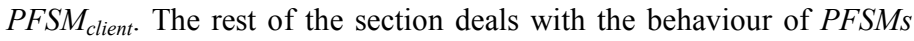
i.e. the transitions associated with each of the PFSMs.

Table 1: List of messages

\begin{tabular}{|c|c|l|}
\hline S.No & Message & \multicolumn{1}{|c|}{ Title } \\
\hline 1 & CR & Connection Request \\
\hline 2 & CI & Connection Indication \\
\hline 3 & CC & Connection Confirmation \\
\hline 4 & BT & Begin Transmission \\
\hline 5 & BP & Begin Presentation \\
\hline 6 & FP & Finished Presentation \\
\hline 7 & FT & Finished Transmission \\
\hline
\end{tabular}

\begin{tabular}{|c|c|l|}
\hline 8 & $\mathrm{OK}$ & OK \\
\hline 9 & $\mathrm{SL}_{0}$ & Send List $\mathrm{L}_{0}$ \\
\hline 10 & $\mathrm{SL}_{1}$ & Send List $\mathrm{L}_{1}$ \\
\hline 11 & $\mathrm{SL}_{2}$ & Send List $\mathrm{L}_{2}$ \\
\hline 12 & $\mathrm{RL}_{0}$ & Receive List $\mathrm{L}_{0}$ \\
\hline 13 & $\mathrm{RL}_{1}$ & Receive List $\mathrm{L}_{1}$ \\
\hline 14 & $\mathrm{RL}_{2}$ & Receive List $\mathrm{L}_{2}$ \\
\hline 15 & $\mathrm{NL}$ & New List \\
\hline 16 & $\mathrm{DR}$ & Disconnection Request \\
\hline 17 & $\mathrm{DI}$ & Disconnection Indication \\
\hline 18 & $\mathrm{DC}$ & Disconnection Confirmation \\
\hline 19 & $\mathrm{PS}$ & Pause \\
\hline 20 & $\mathrm{RS}$ & Resume \\
\hline 21 & $\mathrm{FR}$ & Fast-forward/Rewind \\
\hline 22 & $\mathrm{DS}$ & Discard \\
\hline 23 & $\mathrm{AI}$ & Authoring-based Interaction \\
\hline 24 & $\mathrm{RD}$ & Receive Data \\
\hline 25 & $\mathrm{SP}$ & Start Presentation \\
\hline
\end{tabular}

\section{Client PFSM}

The PFSM at the client side represents the status of the ongoing presentation at any point of time. It is modeled using 3 states $S_{1}, S_{2}$, $S_{3}$ and 18 transitions as detailed below. Figure 1 shows the transition diagram for the PFSM client .

T1: When the request for the presentation is initiated by the user, the system is in state $S_{l}$. This is represented by the Start Presentation (SP) message from the $P F S M_{\text {client }}$ to itself. The $P F S M_{\text {client }}$ initiates the connection establishment further by sending $C R$ messages to $L G$ and remains in state $S_{l}$ until connection has been established successfully.

T2: In state $S_{l}$, when the Connection Indication (CI) message is received from $L G$, a request for the list $L_{0}$ is sent to $L G$.

T3: In state $S_{1}$, when the Receive List $R L_{0}$ message followed by the list $L_{0}$ of all participating media objects is received from $L G$, the $P F S M_{\text {client }}$ further initiates connection establishment ( $C R$ message) with all the MSM in $L_{0}$ and with the PFSM $M_{\text {Server }}$.

T4: In state $S_{1}$ when the Send List $\left(S L_{0}\right)$ is received from the PFSM $_{\text {Server }}$ requesting for the list $L_{0}$, it is forwarded to the $P F S M_{\text {server }}$ preceded by the $R L_{0}$ message. 


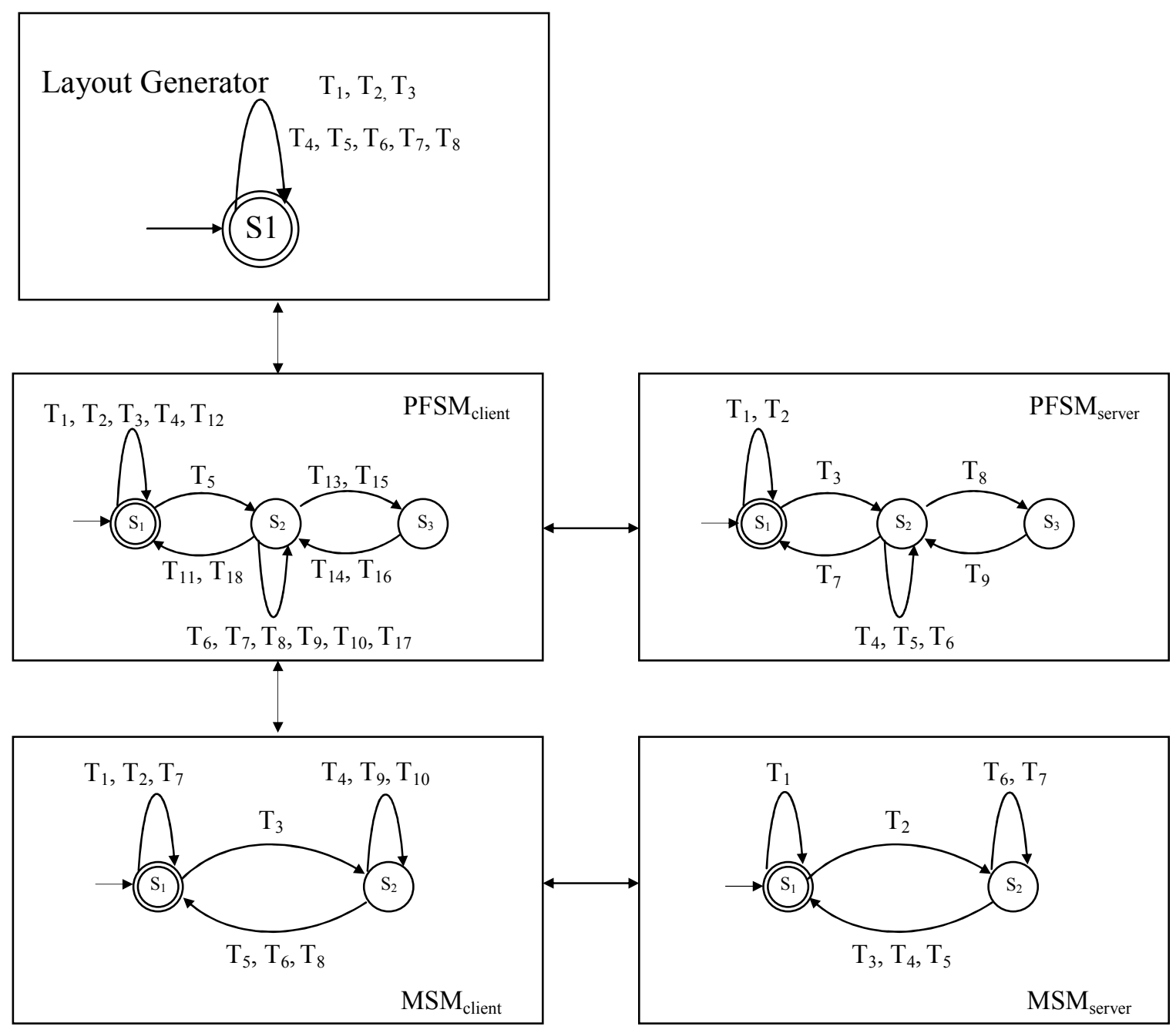

Figure 1: CAFSM Model

T5: In state $S_{l}$, when the Connection Confirmation (CC) message is received from all the MSMs in $L_{0}$ and the $C I$ message is received from the $P F S M_{\text {server }}$, the $P F S M_{\text {client }}$ changes state to $S_{2}$ and sends the Send List $\left(S L_{1}\right.$ and $\left.S L_{2}\right)$ messages to $L G$, requesting for lists $L_{1}$ and $L_{2}$.

T6: In state $S_{2}$, a message $R L_{1}$ from $L G$, indicating that the list $L_{l}$ is being sent, triggers this transition. The lists are received and a Begin Transmission (BT) message is sent to the PFSM server. The list $L_{2}$ could possibly be empty at this stage (for the first synchronization point $L_{2}$ will definitely be empty as no media object ends there).

T7: This transition is triggered in state $S_{2}$, by a message $R L_{2}$ from $L G$, indicating that the list $L_{2}$ is being sent. At this stage the list $L_{l}$ could be possibly empty (for the last synchronization point $L_{l}$ will definitely be empty as no media object begins there).

T8: When in state $S_{2}$ and a $S L_{1}$ message from the $P F S M_{\text {server }}$ requesting for the list $L_{l}$ is received, the available list is forwarded.

T9: As mentioned earlier, $O K$ messages are sent by each $M S M$ as they receive sufficient data in their buffer during streaming. When in state $S_{2}$ and all the $O K$ messages are received from $M S M S$ of media objects that belong to the list $L_{l}$, the Begin Presentation $(B P)$ message is sent to all the media objects that need to 
commence. After sending the $B P$ messages, the List $L_{l}$ is emptied and the request for the new list is sent.

T10: As the Finish Presentation (FP) messages are received in state $S_{2}$ from all the $M S M s$ corresponding to the list $L_{2}$, they are eliminated from the list. When $L_{2}$ becomes empty, the request for the new list is forwarded to $L G$.

T11: At the end of the presentation, when there are no more synchronization points, the LG sends a Disconnection Indication $(D I)$ in response to a request from the $P F S M_{\text {client }}$ for the lists of objects corresponding to the next synchronization point. At this instance, $L_{I}$ and $L_{2}$ are empty and the PFSM client changes state to $S_{1}$ from state $S_{2}$, and continues with the disconnection process by sending disconnection request $(D R)$ messages to the $P F S M_{\text {server }}$ and $\operatorname{MSM}\left(L_{0}\right)$ at the client.

T12: In state $S_{l}$, the $P F S M_{\text {client }}$ waits until it receives the Disconnection Indication $(D I)$ from the $P F S M_{\text {server }}$ and Disconnection Confirmations $(D C)$ from $\operatorname{MSM}\left(L_{0}\right)$ and it remains in $S_{l}$ which is also considered as the final state of the system.

T13: In case the $P F S M_{\text {client }}$ receives a fast-forward/rewind FR message from the user while it is in state $S_{2}$, it sends a Discard Message (DS) to the PFSM server $_{\text {and to }}$ the MSMs of all participating media objects at the client. It also calculates the new index value that represents the point at which the presentation should resume and sends it to $L G$ with a request for a new list that includes the media objects from the point requested by the user and then changes to state $S_{3}$. The lists $L_{1}$ and $L_{2}$ are discarded.

T14: In state $S_{3}$, the $P F S M_{\text {client }}$ receives the $F P$ messages from the $M S M s$ of all media objects at the client, indicating that they have successfully discarded all the previous data. It also receives a $R L_{l}$ message from the $L G$ which then sends a new list $L_{1}$. Subsequently, the $P F S M_{\text {client }}$ sends a $B T$ message to the PFSM server and changes the state to $S_{2}$.

T15: While in $S_{2}$, the $P F S M_{\text {client }}$ could receive an interaction in the form of Pause (PS) Presentation. The PFSM client merely forwards this message to the MSMs of all the media objects involved in the presentation and goes to the state $S_{3}$.

T16: While in $S_{3}$, the $P F S M_{\text {client }}$ could receive an interaction in the form of resume $R S$ message which is normally preceded by a $P S$ message. In this case, the $P F S M_{\text {client }}$ also forwards this message to the $M S M$ of all the media objects involved in the presentation and goes to the state $S_{2}$.

T17: While in $S_{2}$, the $P F S M_{\text {client }}$ could receive an Adaptationbased-Interaction (AI) message and that could result in a change in the presentation layout. In this case, the $P F S M_{\text {client }}$ forwards the data relating to this interaction to the $L G$ along with a request for a New List $(N L)$ message and sends $D S$ messages to the $P F S M_{\text {server }}$ and all its MSMs.

T18: In state $\mathrm{S}_{2}$, when a request for disconnection from the user (represented here by a Disconnection Request (DR) message, in which the PFSM client sends to itself in order to further initiate the disconnection process) is received, it sends $D R$ messages to the $P F S M_{\text {server }}$ and to the $\operatorname{MSM}\left(L_{0}\right)$, and goes back to state $S_{l}$.

\section{Server PFSM}

The PFSM at the server side represents at any point of time the status of the ongoing transmissions. It is modelled using 3 states say $\mathrm{S}_{1}, \mathrm{~S}_{2}$ and $\mathrm{S}_{3}$, and 9 transitions as detailed below. Figure 1 shows the transition diagram for the $P F S M_{\text {server }}$. The transitions in the $P F S M_{\text {server }}$ require only the lists $L^{\prime}$ and $L^{*}$ here contains only list $L_{l}$.

T1: The Connection Request (CR) initiated by the $P F S M_{\text {client }}$ triggers this transition in state $S_{l}$. At this stage, the list $L_{l}$ is empty. It responds with a Send List $S L_{0}$ message to the $P F S M_{\text {client }}$.

T2: The PFSM $M_{\text {client }}$ sends a Receive List $\left(R L_{0}\right)$ message followed by the list $L_{0}$. On receiving this message the PFSM $_{\text {server }}$ sends a $(C I)$ message to the PFSM client , and continues to remain in state $S_{l}$.

T3: When the PFSM $M_{\text {server }}$ is in state $S_{1}$ and it receives Connection Indication (CI) messages from all the $M S M s$, it changes the state to $S_{2}$.

T4: While in state $S_{2}$, the $P F S M_{\text {server }}$ receives Begin Transmission (BT) message from the $P F S M_{\text {client }}$. It remains in the same state and makes a request to the $P F S M_{\text {client }}$ for the list $L_{l}$.

T5: In state $S_{2}$, a Receive List $\left(R L_{1}\right)$ message from the PFSM $M_{\text {client }}$ indicates that the list $L_{l}$ is being sent. A $B T$ message is sent to each MSM in the received list.

T6: While in state $S_{2}$, as the $F T$ messages are received from the MSMs corresponding to list $L_{l}$, they are eliminated from the list and a request for the next list $S L_{1}$ is transmitted.

T7: When in state $S_{2}$, on receiving the Disconnection Request (DR) from PFSM client and Disconnection Indication $(D I)$ from $M S M\left(L_{0}\right)$ at the server, it sends a $D I$ in turn to $P F S M_{\text {client }}$ and changes the state to $S_{l}$.

T8: $\quad$ The $P F S M_{\text {server }}$, on receiving the $D S$ message, moves to state $S_{3}$ and discards the list $L_{1}$.

T9: When in state $S_{3}$, on receiving $F T$ messages from the $M S M s$ of all media objects involved in the presentation, it changes the state to $S_{2}$.

\subsection{Media Schedule Managers : MSM}

Formally, a $M S M$ can be defined as a 3-tuple $(S, \Sigma, \delta)$, where $S$ is a finite set of states $\left\{S_{1}, S_{2}\right\}, \Sigma$ is a set of messages and $\delta: S \times 2^{\Sigma} \rightarrow$ $S \times 2^{\Sigma}$ is a transition function that takes as input the state of the machine and the messages received and changes its state. In the subsequent paragraphs, the transitions associated with client and server MSMs are described. 


\section{Client MSM}

T1: In state $S_{l}$, when the $M S M_{\text {client }}$ receives a request for connection from its PFSM, it forwards the request to its peered $M S M$ at the server.

T2: In state $\mathrm{S}_{1}$, on receiving a Connection Indication (CI) from the $M S M_{\text {server }}$, the $M S M_{\text {client }}$ sends a message confirming the connection to its PFSM. This completes connection establishment.

T3: In state $S_{1}$, on receiving sufficient data (which follows the $R D$ message) from their peered $M S M s$, the $M S M_{\text {client }}$ sends an $O K$ message to the PFSM indicating that it is ready for the presentation, and changes its state to $\mathrm{S}_{2}$.

T4: While in state $\mathrm{S}_{2}$, the $M S M_{\text {client }}$ receives a $B P$ message from the PFSM and it commences its presentation.

T5: While in state $\mathrm{S}_{2}$, after completion of the play out, it generates a message $F P$ to itself which triggers a change of state to $\mathrm{S} 1$ and sends a Finish Presentation (FP) message to the PFSM client.

T6: While in state $\mathrm{S}_{2}$, the $M S M_{\text {client }}$ may receive a Disconnection Request (DR). This will change its state to $S_{I}$ and it will forward the request to its peered $M S M$ at the server.

T7: At state $\mathrm{S}_{1}$, the peered MSM replies with a Disconnection Indication $(D I)$, and the $M S M_{\text {client }}$ in turn sends a Disconnection Confirmation (DC) message to the PFSM $M_{\text {client }}$.

T8: In state $S_{2}$, when the $M S M_{\text {client }}$ receives the Discard message (DS) from the PFSM $M_{\text {client }}$, it changes the state to $S_{1}$ and sends Discard messages $(D S)$ to its peered $M S M_{\text {server }}$. It also sends a FP message to the $P F S M_{\text {client }}$ indicating that it has successfully discarded the current presentation data.

T9: In state $S_{2}$, when the $M S M_{\text {client }}$ receives the Pause (PS) message from the $P F S M_{\text {client }}$, it forwards the message to its peered $M S M_{\text {server }}$ at the server. It also sends a FP message to the PFSM indicating that it has successfully paused the presentation of the media object it represents.

T10: In state $S_{2}$, when the $P F S M_{\text {client }}$ receives a Resume (RS) message, it continues with the presentation of the media object.

\section{Server MSM}

T1: In state $S_{l}$, when the $M S M_{\text {server }}$ receives the $C R$ message from the $M S M_{\text {client }}$, it sends a $C I$ message back to its peered $M S M_{\text {client }}$ and also to the PFSM server .

T2: In state $S_{1}$, on receiving a $B T$ message from $P F S M_{\text {server }}$, the $M S M_{\text {server }}$ changes its state to $S_{2}$ and starts transmitting the data to its peered $M S M_{\text {client }}$.

T3: In state $S_{2}$, at the end of transmission, it triggers itself with a Finish Transmission (FT) message and forwards the FT message, to the $P F S M_{\text {server }}$ and changes the state to $S_{1}$.
T4: When in state $\mathrm{S}_{2}$, if a message requesting disconnection $D R$ is received, then the $M S M_{\text {client }}$ sends a Disconnection Indication (DI) to the $M S M_{\text {client }}$ and to the PFSM server and changes the state to $S_{l}$.

T5: In state $\mathrm{S}_{2}$, when the $M S M_{\text {server }}$ receives a Discard message (DS) from its $M S M_{\text {client }}$, it sends a FT message to the $P F S M_{\text {server }}$ and changes state to $S_{l}$.

T6: In state $S_{2}$, when the $M S M_{\text {server }}$ receives a Pause message (PS) from the peer $M S M_{\text {client }}$, it sends a Finish Transmission (FT) message to the $P F S M_{\text {server }}$ and remains in $S_{2}$

T7: In state $S_{2}$, when the $M S M_{\text {server }}$ receives a Resume message (RS) from its peer $M S M_{\text {client }}$, it continues with its transmission.

\subsection{Layout Generator : LG}

Formally, the layout generator $L G$ can be defined as a 3-tuple $\left(\left\{S_{I}\right\}, \Sigma, \delta\right)$, where $S_{I}$ is its only state, $\Sigma$ is a set of messages, and $\delta$ $: S \times 2^{\Sigma} \rightarrow S \times 2^{\Sigma}$ is a transition function which takes as input the messages received to trigger new messages and perform appropriate actions. The transitions are shown in Figure 1.

T1: When the $L G$ receives the $C R$ message from the $P F S M_{\text {client }}$, it sends a $C I$ message back to the PFSM client.

T2: When the $L G$ receives a $S L_{0}$ message requesting for the lists $L_{0}$, the receive lists $R L_{0}$ message is sent followed by the data (i.e. $L_{0}$ ).

T3: When the $L G$ receives a $S L_{l}$ message requesting for the lists $L_{l}$, the receive lists $R L_{l}$ message is sent followed by the data (i.e. $L_{1}$ ).

T4: When the $L G$ receives a $S L_{2}$ message requesting for the lists $L_{2}$, the receive lists $R L_{2}$ message is sent followed by the data (i.e. $L_{2}$ ).

T5: If the user abruptly ends the presentation, then the $L G$ receives a Disconnection Request from the $P F S M_{\text {client }}$ and sends a Disconnection Indication back to the PFSM $_{\text {client }}$.

T6: On the other hand, if the PFSM client requests for the next set of lists while there are no more synchronization points, then the $L G$ sends a $D R$ messages to itself which triggers further disconnection by sending Disconnection Indication message to the PFSM client

T7: Here, the request from the PFSM for a new list is made following a fast-forward/rewind request made by the user. The PFSM also computes the index value to indicate the location where the presentation should commence, and sends this as the index value $i$. The $L G$ sends a new list replacing the existing list $L_{l}$.

T8: The $P F S M_{\text {client }}$ requests for the next set of lists in the form of the message new list $N L$, followed by data. This represents an (adaptation-based) interaction with 
the presentation which could lead to changes in the spatiotemporal relations and may cause inconsistency to occur. Thus, the consistency checking algorithm [6] is employed by the $L G$ to produce a consistent set of constraints with the $L G$ using the modified layout to generate the next list $L_{l}$ as data to the PFSM client.

\section{ANALYSIS OF THE PROPOSED MODEL}

The salient feature of the proposed model is the design of the set of transitions that guide the runtime module to ensure a synchronised playout of the presentation. The set of transitions, presented along with each of the CAFSMs in the previous section, also define the sequence of the messages that are used to control the flow of the presentation. The proposed model is deterministic i.e there is only one path that can be traversed for a particular event. For multimedia presentations that have rapidly changing objects, there would be several synchronisation points leading to several states and transitions in the existing DEFSM model, while the proposed CAFSMs have a fixed number of states and transitions irrespective of the number of synchronisation points. The response time, in case of interactions with the presentation as well as navigations like skip, would then have a worst-case complexity of $O(n)$, where $n$ represents the number of transitions to search from in the existing DEFSM models. But in the proposed CAFSM model, there are only 3 states and 18 transitions (at the most) to search from at any point of time, leading to a system with an efficient response time to interactions. The proposed CAFSM model has however been checked using the model checker SPIN and the simulation results obtained have correlated with the theoretical results.

\section{CONCLUSIONS}

This paper presents the design of a multimedia synchronisation mechanism using a formal approach. The proposed CAFSM model with the help of its message passing scheme ensures a synchronised play out of the presentation and is also efficient in its time and space complexity in comparison with the existing formal approaches that define similar synchronization mechanisms. The proposed model can be further enhanced to handle adaptive presentations, buffer management and efficient retrieval policies for bandwidth restricted mobile multimedia communication.

\section{ACKNOWLEDGEMENT}

We would like to acknowledge and thank the Defence Research Development Organisation (DRDO), New Delhi, India for granting us Extramural Research Funds for carrying out this research work. This work is part of the research project titled "The design and development of a multimedia presentation system that streams MPEG-21 compatible media-on-demand".

\section{REFERENCES}

[1] E. Bertino and E. Ferrari. Temporal synchronization models for multimedia data. IEEE Trans. on Knowl. and Data Eng., 10(4):612-631, 1998.

[2] D. Brand and P. Zafiropulo. On communicating finite-state machines. J. ACM, 30(2):323-342, 1983.

[3] J. Canavan. Personalised e-learning through learning style aware adaptive systems: MS thesis, 2004.

[4] H. Chorfi and M. Jemni. Perso: Towards an adaptive elearning system. Journal of Interactive Learning Research, 15(4):433-447, 2004.

[5] O. Conlan and V. Wade. Evaluation of APeLS - an adaptive eLearning service based on the multi-model, metadata- driven approach. Adaptive Hypermedia and Adaptive Web-Based Systems, 3137:291-295, 2004.

[6] S. Elias, K. S. Easwarakumar, and R. Chbeir. Dynamic consistency checking for temporal and spatial relations in multimedia presentations. In Proceedings of The 21st ACM Symposium on Applied Computing, pages 1380-84, Dijon, France, April 2006.

[7] T. Flor. Experiences with adaptive user and learning models in elearning systems for higher education. Journal of Universal Computer Science, 10(1):58-72, 2004.

[8] C. Gütl, M. Pivec, C. Trummer, V. M. García-Barrios, F. Modritscher, J. Pripfl, and M. Umgeher. Adele (adaptive e-learning with eye-tracking): Theoretical background, system architecture and application scenarios. European Journal of open, Distance and Elearning(EURODL), 2004.

[9] C.-M. Huang and C. Wang. Synchronization for interactive multimedia presentations. IEEE Multimedia, pages 44-61, Oct. 1998.

[10] C. M. Huang, C. Wang, and C. H. Lin. Interactive multimedia synchronisation in the distributed environment using the formal approach. IEE Proceedings - Software, 147(4):131-146, 2000.

[11] M. Kock. Computational intelligence for communication and cooperation guidance in adaptive e-learning systems. In $L W A$, volume 448 of Technical Report, pages 32-34. Department of Computer Science, University of Wurzburg, Germany, 2008.

[12] F. Modritscher, V. Garcia Barrios, and C. Gutl. Enhancement of scorm to support adaptive e-learning within the scope of the research project adele. In Proceedings of World Conference on E-Learning in Corporate, Government, Healthcare, and Higher Education 2004, pages 2499-2505, Washington, DC, USA, 2004.

[13] M. Meccawy, P. Brusilovsky, H. Ashman, M. Yudelson, and O. Scherbinina. Integrating interactive learning content into an adaptive e-learning system: Lessons learned. In Proceedings of World Conference on E-Learning in Corporate, Government, Healthcare, and Higher Education 2007, pages 6314-6319, Quebec City, Canada, 2007.

[14] H. D. Surjono. Empirical evaluation of an adaptive elearning system and the effects of knowledge, learning styles and multimedia mode on student achievement. In Proceedings of the UiTM International Conference on E-Learning, pages 12-14, Shah Alam, Malaysia, December 2007

[15] C.-C. Yang, Y.-C. Wang, and C.-W. Tein. Synchronization modeling and its application for SMIL 2.0 presentations. The journal of Systems and Software, 80:1142-1155, 2007.

[16] A. Zhang. Synchruler: A rule-based flexible synchronization model with model checking. IEEE Trans. on Knowl. and Data Eng., 17(12):1706-1720, 2005. Member-Aygun, Ramazan Savas.

[17] www.engineeringchallenges.org

[18] Mödritscher, F., Garcia Barrios, V.M. \& Gütl, C. (2004). Enhancement of SCORM to support adaptive E-Learning within the Scope of the Research Project AdeLE. In Proceedings of World Conference on ELearning in Corporate, Government, Healthcare, and 
Higher Education 2004 (pp. 2499-2505). Chesapeake, VA:

AACE.

\section{APPENDIX}

\section{A. CLIENT PFSM}

T1: $\delta\left(S_{l},\left(S P, P F S M_{\text {client }}\right), \phi, \phi\right)=\left(S_{1},(C R, L G), \phi, \phi\right)$

T2: $\left.\delta\left(S_{1},\{(C I, L G)\}, \phi, \phi\right)=\left(S_{1},\left(S L_{0}\right), L G\right), \phi, \phi\right)$

T3: $\delta\left(S_{1},\left\{\left(R L_{0}, L G\right), L_{0}, \phi\right)=\left(S_{1},\left\{\left(C R, P F S M_{\text {server }}\right),\left(C R, M S M\left(L_{0}\right)\right)\right\}, \phi, \phi\right)\right.$

T4: $\delta\left(S_{1},\left(S L_{0}, P F S M_{\text {server }}\right), \phi, \phi\right)=\left(S_{1},\left\{\left(R L_{0}, P F S M_{\text {server }}, L G\right),\left(S L_{2}, L G\right)\right\}, L_{0}, \phi\right)$

T5: $\delta\left(S_{1},\left\{\left(C C, M S M\left(L_{0}\right)\right),\left(C I, P F S M_{\text {Server }}\right)\right\}, \phi, \phi\right)=\left(S_{2},\left\{\left(S L_{1}, L G\right),\left(S L_{2}, L G\right)\right\}, \phi, \phi\right)$

T6: $\delta\left(S_{2},\left(R L_{1}, L G\right), \phi, L_{2}\right)=\left(S_{2},\left(B T, P F S M_{\text {Server }}\right), L_{1}, L_{2}\right)$

T7: $\delta\left(S_{2},\left(R L_{2}, L G\right), L_{1}, \phi\right)=\left(S_{2}, \phi, L_{1}, L_{2}\right)$

T8: $\delta\left(S_{2},\left(S L_{1}, P F S M_{\text {Server }}\right), L_{1}, L_{2}\right)=\left(S_{2},\left(R L_{1}, P F S M_{\text {Server }}\right), L_{1}, L_{2}\right)$

T9: $\delta\left(S_{2},\left(O K, M S M\left(L_{1}\right), L_{1}, L_{2}\right)=\left(S_{2},\left\{\left(B P, M S M\left(L_{1}\right),\left(S L_{1}, L G\right)\right\}, \phi, L_{2}\right)\right.\right.$

T10: $\delta\left(S_{2},\left(F P, M S M\left(L_{2}\right)\right), L_{1}, L_{2}\right)=\left(S_{2},\left(S L_{2}, L G\right), L_{1}, \phi\right)$

T11: $\delta(S 2,(D I, L G), \phi, \phi)=\left(S_{1},\left\{\left(D R, P F S M_{\text {Server }}\right),\left(D R, M S M\left(L_{0}\right)\right\}, \phi, \phi\right)\right.$

T12: $\delta\left(\mathrm{S}_{1},\left\{\left(D I, P F S M_{\text {Server }}\right),\left(D C, M S M\left(L_{0}\right)\right\}, \phi, \phi\right)=\left(S_{1}, \phi, \phi, \phi\right)\right.$

T13: $\delta\left(S_{2},\left(F R, P F S M_{\text {Client }}\right), L_{1}, L_{2}\right)=\left(S_{3},\left\{\left(D S, P F S M_{\text {Server }}\right),\left(D S, M S M\left(L_{0}\right),((N L, i), L G)\right\}, \phi, \phi\right)\right.$

T14: $\delta\left(S_{3},\left\{\left(F P, M S M\left(L_{0}\right),\left(R L_{1}, L G\right)\right\}, \phi, \phi\right)=\left(S_{2},\left(B T, P F S M_{\text {Server }}\right), L_{1}, \phi\right)\right.$

T15: $\delta\left(S_{2},\left(P S, P F S M_{\text {client }}\right), L_{1}, L_{2}\right)=\left(S_{3},\left\{\left(P S, M S M\left(L_{0}\right)\right),\left(P S, P F S M_{\text {Server }}\right)\right\}, \phi, \phi\right)$

T16: $\delta\left(S_{3},\left(R S, P F S M_{\text {client }}\right), L_{1}, L_{2}\right)=\left(S_{2},\left\{\left(R S, P F S M_{\text {Server }}\right),\left(R S, M S M\left(L_{0}\right)\right)\right\}, \phi, \phi\right)$

T17: $\delta\left(S_{2},\left(A I, P F S M_{\text {client }}\right), L_{1}, L_{2}\right)=\left(S_{2},\left\{\left(D S, P F S M_{\text {server }}\right),\left(D S, M S M\left(L_{0}\right),((N L\right.\right.\right.$, data $\left.\left.), L G)\right\}, \phi, \phi\right)$

T18: $\delta\left(S_{2},\left(D R, P F S M_{\text {client }}\right), L_{1}, L_{2}\right)=\left(S_{1},\left\{\left(D R, P F S M_{\text {server }}\right),\left(D R, M S M\left(L_{0}\right)\right),(D R, L G)\right\}, \phi, \phi\right)$

\section{B. SERVER PFSM}

T1: $\delta\left(S_{l},\left(C R, P F S M_{\text {client }}\right), \phi\right)=\left(S_{1},\left(S L_{0}, P F S M_{\text {Client }}\right), \phi\right)$

T2: $\delta\left(S_{1},\left(R L_{0}, P F S M_{\text {client }}\right), L_{0}\right)=\left(S_{l}, \phi, \phi\right)$

T3: $\delta\left(S_{1},\left(C I, M S M\left(L_{0}\right)\right), \phi\right)=\left(S_{2},\left(C I, P F S M_{\text {client }}\right), \phi\right)$

T4: $\delta\left(S_{2},\left(B T, P F S M_{\text {client }}\right), \phi\right)=\left(S_{2},\left(S L_{1}, P F S M_{\text {client }}\right), \phi\right)$

T5: $\delta\left(S_{2},\left(R L_{1}, P F S M_{\text {client }}\right), \phi\right)=\left(S_{2},\left(B T, M S M_{L l}\right), L_{1}\right)$

T6: $\delta\left(S_{2},\left(F T, M S M\left(L_{1}\right)\right), L_{1}\right)=\left(S_{2},\left(S L_{1}, P F S M_{\text {client }}\right), \phi\right)$

T7: $\delta\left(S_{2},\left\{\left(D R, P F S M_{\text {client }}\right),\left(D I, M S M\left(L_{0}\right)\right)\right\}, \phi\right)=\left(S_{1},\left(D I, P F S M_{\text {client }}\right), \phi\right)$

T8: $\delta\left(S_{2},\left(D S, P F S M_{\text {client }}\right), L_{1}\right)=\left(S_{3}, \phi, \phi\right)$

T9: $\delta\left(S_{3},\left(F T, M S M\left(L_{0}\right)\right), \phi\right)=\left(S_{2}, \phi, \phi\right)$

\section{CLIENT MSM}

T1: $\delta\left(S_{l},\left(C R, P F S M_{\text {client }}\right)\right)=\left(S_{l},\left(C R, M S M_{\text {server }}\right)\right)$

T2: $\delta\left(S_{1},\left(C I, M S M_{\text {server }}\right)\right)=\left(S_{1},\left(C C, P F S M_{\text {client }}\right)\right)$

T3: $\delta\left(S_{1},\left(\right.\right.$ Data $_{1}$ MSM $\left._{\text {server }}\right)=\left(S_{2},\left(O K, P F S M_{\text {client }}\right)\right)$

T4: $\delta\left(S_{2},\left(B P, P F S M_{\text {client }}\right)\right)=\left(S_{2}, \phi\right)$

T5: $\delta\left(S_{2},\left(F P, M S M_{\text {client }}\right)\right)=\left(S_{l},\left(F P, P F S M_{\text {client }}\right)\right)$

T6: $\delta\left(S_{2},\left(D R, P F S M_{\text {client }}\right)\right)=\left(S_{1},\left(D R, M S M_{\text {server }}\right)\right)$

T7: $\delta\left(S_{l},\left(D I, M_{S M} M_{\text {server }}\right)\right)=\left(S_{1},\left(D C, P_{1}\right.\right.$ S $\left.\left._{\text {client }}\right)\right)$

T8: $\delta\left(S_{2},\left(D S, P F S M_{\text {client }}\right)\right)=\left(S_{1},\left\{\left(D S, M S M_{\text {server }}\right),\left(F P, P F S M_{\text {client }}\right)\right\}\right)$

T9: $\delta\left(S_{2},\left(P S, P F S M_{\text {client }}\right)\right)=\left(S_{2},\left\{\left(P S, M S M_{\text {server }}\right),\left(F P, P F S M_{\text {client }}\right)\right\}\right)$

T10: $\delta\left(S_{2},\left(R S, P F S M_{\text {client }}\right)\right)=\left(S_{2},\left(R S, M S M_{\text {server }}\right)\right)$

\section{SERVER MSM}

T1: $\delta\left(S_{l},\left(C R, M S M_{\text {client }}\right)\right)=\left(S_{l},\left\{\left(C I, M S M_{\text {client }}\right),\left(C I, P F S M_{\text {server }}\right)\right\}\right)$

T2: $\delta\left(S_{1},\left(B T, P F S M_{\text {server }}\right)\right)=\left(S_{2},\left(R D, M S M_{\text {Client }}\right)\right)$

T3: $\delta\left(S_{2},\left(F T, M_{S M_{\text {server }}}\right)\right)=\left(S_{1},\left(F T, P F S M_{\text {server }}\right)\right)$

T4: $\delta\left(S_{2},\left(D R, M S M_{\text {client }}\right)\right)=\left(S_{1},\left\{\left(D I, M S M_{\text {client }}\right),\left(D I, P F S M_{\text {server }}\right)\right\}\right)$

T5: $\delta\left(S_{2},\left(D S, M S M_{\text {client }}\right)\right)=\left(S_{1},\left(F T, P F S M_{\text {server }}\right)\right)$

T6: $\delta\left(S_{2},\left(P S, M S M_{\text {client }}\right)\right)=\left(S_{2},\left(F T, P F S M_{\text {server }}\right)\right)$

T7: $\delta\left(S_{2},\left(R S, M S M_{\text {client }}\right)\right)=\left(S_{2},\left(\right.\right.$ Data,$\left.\left.M S M_{\text {client }}\right)\right)$

\section{E. LAYOUT GENERATOR}

T1: $\delta\left(S_{l},\left\{\left(C R, P F S M_{\text {client }}\right)\right\}\right)=\left(S_{l},\left(C I, P F S M_{\text {client }}\right)\right)$

T2: $\delta\left(S_{l},\left\{\left(S L_{0}, P F S M_{\text {client }}\right\}\right)=\left(S_{l},\left(R L_{0}, P F S M_{\text {client }}\right)\right)\right.$

T3: $\delta\left(S_{l},\left\{\left(S L_{1}, P F S M_{\text {client }}\right\}\right)=\left(S_{l},\left(R L_{1}, P F S M_{\text {client }}\right)\right)\right.$

T4: $\delta\left(S_{1},\left\{\left(S L_{2}, P F S M_{\text {client }}\right\}\right)=\left(S_{1},\left(R L_{2}, P F S M_{\text {client }}\right)\right)\right.$

T5: $\delta\left(S_{l},\left\{\left(D R, P F S M_{\text {client }}\right)\right\}\right)=\left(S_{l},\left(D I, P F S M_{\text {client }}\right)\right)$

T6: $\delta\left(S_{l},\{(D R, L G)\}\right)=\left(S_{l},\left(D I, P F S M_{\text {client }}\right)\right)$

T7: $\delta\left(S_{l},\left((N L, i), P F S M_{\text {client }}\right)\right)=\left(S_{l},\left(R L_{1}, P F S M_{\text {client }}\right)\right)$

T8: $\delta\left(S_{1},((N L\right.$, data $\left.), P F S M)\right)=\left(S_{1},\left(R L_{1}, P F S M_{\text {clien }}\right)\right)$

T9: $\delta\left(S_{1},((N L\right.$, data $\left.), P F S M)\right)=\left(S_{1},\left(R L_{1}, P_{F S M} M_{\text {client }}\right)\right)$ 